\title{
Prospective Evaluation of the Use of Lokivetmab in Dogs With Atopic Dermatitis in Brazil
}

\section{Millena Leme Campos ( $\nabla$ micampos.vet@gmail.com )}

Caopanheiro Clinic

\section{Ronaldo Lucas}

Dermatoclinica

Jonas Moraes-Filho

UNISA/SP

Viviani di Marco

Naya Especialities

Jessica Dinelli Lopes

Dermatoclinica

Daniela Beviani

Dermatoclinica

Carla Pelegrini

Dermatoclinica

\section{Vitoria Miranda Carrao Cunha}

Dermatoclinica

\section{Research Article}

Keywords: Atopic dermatitis, dog, IL-31, lokivetmab, pruritus

Posted Date: October 20th, 2021

DOI: https://doi.org/10.21203/rs.3.rs-916691/v1

License: (c) (i) This work is licensed under a Creative Commons Attribution 4.0 International License.

Read Full License 


\section{Abstract}

Background: Lokivetmab (Cytopoint $\circledast$, Zoetis) is a caninized anti-IL-31 monoclonal antibody licensed for treating clinical manifestations of canine atopic dermatitis in varying degrees of severity. The objective of this 30-day prospective study was to evaluate the level of improvement in cases of atopic dermatitis after the application of lokivetmab, using the Canine Atopic Dermatitis Extent and Severity Index (CADESI-4) variables, Visual Analogue Scale (VAS) of pruritus, owner assessment of the condition, and incidence of adverse events. A total of 110 animals recruited from two private clinics in the State of São Paulo each received $2 \mathrm{mg}$ of lokivetmab/ $\mathrm{kg}$ of body weight by subcutaneous injection on day 0 . Dogs were observed by owners who provided reports of their observations by telephone or e-mail on days 2 and 14 and were observed by clinicians on day 30 .

Results: Baseline mean VAS of pruritus was 7.68 (median $=7$ ), decreasing to 3.82 (median $=2$ ) by day 2 $(P<0.001)$. CADESI-4 variables decreased from 36.5 at baseline to 16.3 on day $30(P<0.001)$. Reports of treatment responses submitted by owners and evaluation of CADESI-4 variables after treatment were significantly similar $(P<0.001)$. Owners rated the response to treatment as good to excellent for $75 \%$ of the dogs. Adverse events, including vomiting, diarrhea, and/or lethargy, were observed in 18 dogs (16.4\%). All events were transient, and none required treatment.

Conclusions: Based on findings in this study, lokivetmab was determined to provide a rapid and effective response for control of canine atopic dermatitis. Lokivetmab was well tolerated with only mild and transient adverse events observed.

\section{Background}

Atopic dermatitis $(A D)$ is a chronic allergic and inflammatory disease, associated with cytokines. The age of onset is generally around 6 months to 3 years of age, and multiple breeds have a genetic predisposition to $A D$, with the incidence within those breeds variable according to the genetic pool and the region [1]. Pruritus is the primary characteristic of the condition, with secondary lesions occurring around the face, ears, ventral abdomen, flexor aspects of elbow, carpal, and tarsal joints, interdigital skin, and perineal area [2-5]. It is caused by different allergens called atopens and can be controlled but cannot be cured [6].

Dogs react to the condition in several ways, including scratching, biting, licking, or rubbing. If intense, the itching often leads to episodes of self-trauma [3, 7]. The Visual Analogue Scale (VAS) of pruritus created by Rybnicek et al [8] is a vertical line with descriptions of the levels of symptom severity ranging from 0 to 10. The scale is applied based on the dog owner's assessment of the level of pruritus.

Interleuken-31 (IL-31) is responsible for inducing pruritus and plays an important role in the pathogenesis of canine AD [4, 9]. In one evaluation of several antipruritic therapeutics in an IL-31-induced model, it was possible to determine relative speed of onset of reduced IL-31-induced pruritis as well as duration of action [10]. Neutralization of IL-31 with a monoclonal antibody (mAb) has been found to provide relief 
from itching related to $A D$ and inflammation, with a greater margin of safety than for less targeted therapies such as corticosteroids or cyclosporine [10-12].

Lokivetmab is a caninized mAb that binds selectively and neutralizes IL-31 $[4,10,13]$. In one study, a significant reduction in pruritus was observed for at least 1 month following administration of a single subcutaneous (SC) injection of lokivetmab at $0.5 \mathrm{mg} / \mathrm{kg}$ or $2 \mathrm{mg} / \mathrm{kg}$ compared to placebo $(P<0.05)$, and the level of response duration increased with additional doses [4]. In a clinical trial in client-owned dogs with $A D$, monthly administration of lokivetmab at $2 \mathrm{mg} / \mathrm{kg} \mathrm{SC}$ (actual doses ranged from 1.0-3.3 mg/kg) was noninferior to daily administration with oral cyclosporine at $5 \mathrm{mg} / \mathrm{kg}$ for pruritus reduction [13]. Mean Canine Atopic Dermatitis Extent and Severity Index (CADESI-03) scores were not significantly different between the treatments at any time points [13]. The benefits of this mAb therapy include its rapid onset of activity, infrequent dosing, lack of age restriction for its use, safety, and compatibility with other drugs $[11,14]$. Lokivetmab is approved in several countries for treatment of canine AD at a minimum dose of 1 or $2 \mathrm{mg} / \mathrm{kg} \mathrm{SC}$, and with expected effectiveness for at least 1 month $[11,15,16]$.

The objective of the present study was to evaluate the efficacy of lokivetmab ( $2 \mathrm{mg} / \mathrm{kg})$ in the control of canine $A D$ through the clinical evolution of lesions using CADESI-4 and the reduction of pruritus measured by VAS. The safety assessment included recording of all adverse events. Dog owners also provided their perception of the treatment response.

\section{Results}

\section{Efficacy}

A total of 110 animals, 1 to 16 years of age met the inclusion criteria (mean $\pm S D=6.73 \pm 3.41$ years; median $=7$ years). Sixty-six animals $(60.0 \%)$ were female and $44(40.0 \%)$ were male. Weight ranged from 2.5 to $39.8 \mathrm{~kg}$ (mean $\pm \mathrm{SD}=13.84 \pm 9.86 \mathrm{~kg}$; median $=9.20 \mathrm{~kg}$ ). Seventeen dogs $(15.5 \%)$ were mixed breeds and 93 (84.6\%) were purebreds (Fig. 1).

The majority of dogs (96.4\%) had previously been treated with other antipruritic medications, without satisfactory success. Treatments given for control of itching and inflammation as reported by the owners are shown in Fig. 2. The medications reported with the highest frequency were deflazacort, oclacitinib, prednisolone, and cyclosporine. Some dogs had received 2 or more medications.

A total of 4 dogs were withdrawn during the study. Reasons for withdrawal included diagnosis with giardiasis, emergency orthopedic surgery, onset of an adverse event, and worsening of pruritus (at the tutor's request).

VAS data are shown in Table 1. The baseline mean VAS score was 7.68 and decreased by approximately $50 \%$ to 3.82 at day $2(P<0.001)$. Scores on days 14 and 30 remained significantly lower than those on day $0(P<0.001)$. Median scores followed a similar pattern, ranging from 7 on day 0 to 3 on days 14 and 30 . 
Table 1

VAS scores for dogs with atopic dermatitis treated with lokivetmab $2 \mathrm{mg} / \mathrm{kg}$

\begin{tabular}{|llllll|}
\hline Study Day & N & Mean & SD & Minimum & Maximum \\
\hline 0 & 106 & 7.68 & 1.10 & 4 & 10 \\
\hline 2 & 106 & $3.82 *$ & 2.46 & 0 & 10 \\
\hline 14 & 106 & $3.15^{*}$ & 2.25 & 0 & 10 \\
\hline 30 & 106 & $3.89 *$ & 4.83 & 0 & 45 \\
\hline \multicolumn{4}{l}{ SD, standard deviation; VAS; visual analog scale } \\
\hline \multicolumn{4}{|l}{ *Significantly different from VAS score on day 0 $(P<0.001)$} \\
\hline
\end{tabular}

The mean CADESI-4 score on day 30 (16.3) was less than half of the baseline mean score (36.5), a statistically significant reduction $(P<0.001)$ (Table 2$)$. There was also a similar reduction in the median score from 25.5 on day 0 to 10.5 on day 30.

Table 2

CADESI-4 scores for dogs with atopic dermatitis treated with lokivetmab $2 \mathrm{mg} / \mathrm{kg}$

\begin{tabular}{|llllll|}
\hline Study Day & $\mathbf{n}$ & Mean & SD & Minimum & Maximum \\
\hline 0 & 106 & 36.5 & 26.1 & 0 & 145 \\
\hline 30 & 106 & $16.3^{*}$ & 16.4 & 0 & 84 \\
\hline
\end{tabular}

There was a positive and significant correlation $(P<0.001)$ for the change from baseline for VAS and CADESI-4 scores (Spearman's $r=0.593$ ) (Fig. 3). Overall, 75\% of owners rated the efficacy of treatment good to excellent (Fig. 4).

Bacterial foliculitis with Staphylococcus spp were diagnosed in 38 dogs (34.5\%) at baseline (Table 3), and 21 of the total population (19.8\%) were positive for Staphylococcus spp on day 30 ( $P=0.024$ compared with day 0 ), which represents $55.3 \%$ of the 38 dogs positive for Staphylococcus spp on day 0. A total of 7 dogs $(6.7 \%)$ negative for any bacterial or fungal skin infection on day 0 developed infection with Malassezia spp during the study. Also, 2 additional dogs (1.9\%) that were negative for any skin infection on day 0 were positive for mixed infection with Staphylococcus spp and Malassezia spp on day 30 . 
Table 3

Number and percentage of dogs with superficial skin infections at baseline and 30 days after treatment with lokivetmab $2 \mathrm{mg} / \mathrm{kg} \mathrm{SC}$

\begin{tabular}{|lllll|}
\hline & \multicolumn{2}{c}{ Day $\mathbf{0}(\mathbf{N}=110)$} & \multicolumn{2}{c|}{ Day $\mathbf{3 0}(\mathbf{N}=106)$} \\
\hline Infection & $\mathbf{n}$ & $\%$ & $\mathbf{n}$ & $\%$ \\
\hline Staphylococcus spp & 38 & 34.5 & 21 & 19.8 \\
\hline Malassezia spp & 0 & 0.0 & 7 & 6.7 \\
\hline Staphylococcus/Malassezia & 0 & 0.0 & 2 & 1.9 \\
\hline Total & 38 & 34.5 & 30 & 28.3 \\
\hline SC, suabcutaneous & & & & \\
\hline
\end{tabular}

\section{Safety}

Adverse events, including vomiting, diarrhea, and/or lethargy, were observed in 18 dogs (16.6\%) following treatment with lokivetmab at $2 \mathrm{mg} / \mathrm{kg} \mathrm{SC}$, with 8 dogs (7.4\%) experiencing an event on the day of treatment, $2(1.9 \%)$ reported the next day, and 8 (7.4\%) reported on day 2 (Table 4$)$. The events were transient and considered to be mild, without the need for additional support treatment.

Table 4

Adverse events reported in dogs treated with lokivetmab $2 \mathrm{mg} / \mathrm{kg}$ day $0(\mathrm{~N}=108)$

\begin{tabular}{|ll|}
\hline $\begin{array}{l}\text { Adverse events occurring } \\
\text { D0-D2 }\end{array}$ & No of dogs (\%) \\
\hline Lethargy & $10(9.3)$ \\
\hline Vomiting & $4(3.7)$ \\
\hline Diarrhea & $2(1.9)$ \\
\hline Vomiting + lethargy & $1(0.9)$ \\
\hline Vomiting + lethargy + diarrhea & $1(1.9)$ \\
\hline Total & $18(16.6)$ \\
\hline
\end{tabular}

\section{Discussion}

Of the 110 dogs diagnosed with atopic dermatitis in the study, the majority (84.5\%) were of a defined breed. This finding is consistent with previously published data [7, 17], showing that dogs of many defined breeds are genetically predisposed to developing atopic dermatitis, likely due to a combination of 
genetic characteristics as well as certain environmental factors [3]. The most frequent breed represented in the present study was shih tzu, which is the most popular dog breed in Brazil [18]. Body weight of the animals ranged from 2.5 to $39.8 \mathrm{~kg}$ (mean $13.8 \mathrm{~kg}$ ), with more than half of the dogs each weighing between 2.5 and $10 \mathrm{~kg}$, demonstrating a current predominance of miniature and small client-owned dogs in Brazil [18]. Approximately $60 \%$ of the dogs in this study were females. Earlier studies do not predict a predisposition for the disease according to sex $[5,13]$. Although there were more female dogs than male dogs presented with atopic dermatitis in the present study, no conclusions were drawn regarding this observation.

A limitation of this study is the absence of a control group. Initially, the study was to be carried out as a randomized clinical trial with a test group (lokivetmab) and a negative (placebo) or positive (corticosteroids) control group. However, with regard to a negative control group, we faced an ethical dilemma when we determined there was a severe degree of pruritus and lesions from itching and rubbing. Thus, it was decided that it would be very difficult to convince the owners that their dog might only receive a placebo for its severe infection. The other option of using a corticosteroids as a positive control treatment could be problematic for dogs with a long history of using prednisolone or other steroid treatments associated with adverse reactions.

Based on American laboratory dose titration and clinical dose determination studies with different dosages [4, 12], the minimum effective dosage was determined to be $2 \mathrm{mg} / \mathrm{kg}$. Pharmacokinetic studies also predicted the $2 \mathrm{mg} / \mathrm{kg}$ dose would have $\mathrm{mAb}$ concentrations above $\mathrm{EC}_{50}$ for 28 days [12]. In the present study, enrollment of animals with more severe AD (VAS $\geq 7$ and CADESI- $4 \geq 20$ ) provided an opportunity to evaluate the level of efficacy over the 30-day period for these cases, noting that many other therapeutic options do not provide prolonged efficacy, as demonstrated by the daily administration of cyclosporine over 28 days of evaluation, compared with a single SC administration of lokivetmab on day 0 [13]. The 30-day efficacy period also provides a sensible, convenient period for dog owners to increase treatment adherence and compliance.

The time it takes for treatment to demonstrate its effect is an extremely important factor. In the present study, the mean VAS on day 0 was 7.68, which demonstrated a significant reduction within 2 days after treatment, remaining stable at the improved level throughout the study. Similarly, there was a satisfactory reduction in severity of lesion scores. CADESI- 4 scores at baseline were reduced by $55 \%$ on day 30 , in agreement with the study by Michels et al [4] where CADESI-4 scores were improved as early as 7 days after treatment.

There was relatively high variability among the animals in the severity of lesions at baseline. Despite the heterogeneity of lesion severity, the enrollment criteria required each dog to have a CADESI-4 score $\geq 20$; therefore, none of the dogs had a skin condition on day 0 that would be considered mild. When evaluating Spearman's correlation between VAS and CADESI-4, there was a positive and significant correlation between these 2 variables, which indicates that as the pruritus improved, so did the skin lesions. This phenomenon probably occurs due to an indirect anti-inflammatory effect of lokivetmab 
which, despite specifically blocking IL-31 (pruritic) rather than targeting other agents of the inflammatory cascade, its role in reducing self-trauma from scratching is reduction of inflammation. This therapeutic aspect of lokivetmab was also highlighted by Moyaert et al. [13].

Associated with clinical improvements, there was also a reduction in Staphylococcus spp infections present on day 30. However, 7 dogs developed Malassezia spp infections despite the use of the antiseptic shampoos, and 2 animals developed a mixed infection of Staphyloccocus spp and Malassezia spp). Lokivetmab treatment appeared to facilitate a reduction of the bacterial infection, but not for the fungal infection. The reduction in bacterial infections was likely due to the reduction in the act of scratching, but no a plausible explanation for the appearance of Malassezia infections. Future studies will include evaluations to attempt to determine the role of $A D$ and treatment, if any, in the development of these fungal infections.

The adverse events observed following administration of lokivetmab were mild and required no additional treatment or drug intervention. Of the 4 patients that were withdrawn from the present study by the owner, only 1 was due to diarrhea and severe vomiting on day 0 , following administration of lokivetmab. The appearance and incidence of vomiting, diarrhea, or lethargy reported in the present study are similar to those reported previously in randomized clinical trials of efficacy and safety of lokivetmab in client-owned dogs, including one large safety study in dogs with reported comorbidities and only minimal restrictions for concurrent medications during the study $[4,13,14]$. As stated in the conclusions of the researchers in those earlier controlled studies, we found that lokivetmab demonstrated a good safety profile when administered by SC injection to dogs with atopic dermatitis.

OGATE is a relevant, but subjective, tool in assessing adherence and success of the therapeutic regimen [19]. In our sample, $75 \%$ of the dog owners rated the treatment as good to excellent, indicating a high level of satisfaction. Despite the majority of positive responses and the significant clinical improvement of pruritus and lesion scores achieved in this study, flares related to exposure to allergens are common and may cause some owners to feel that treatment did not provide the expected response [17]. In their development of a core outcome set for evaluating and comparing outcomes of treatments administered to dogs with atopic dermatitis, Olivry and others acknowledge the subjective nature of OGATE, but advise that it is a useful criterion when assessed in concert with results of clinical assessments [19].

It may be possible to provide acceptable efficacy, perhaps with even fewer adverse reactions, with lower dosages of lokivetmab. Results of one clinical study indicated that lokivetmab at $0.5 \mathrm{mg} / \mathrm{kg}$ provided significant reductions in lesion scores, although the treatment effect was observed later, and the duration of efficacy was shorter than for the dogs treated with lokivetmab at $2 \mathrm{mg} / \mathrm{kg}$ [4]. Future studies may be undertaken to determine whether lower doses will provide adequate efficacy against the clinical effects of AD for dogs in Brazil.

\section{Conclusion}


Lokivetmab administered SC at $2 \mathrm{mg} / \mathrm{kg}$ to dogs with AD was effective in controlling signs and symptoms of canine $A D$, providing significant improvement of pruritus and clinical lesions and high level of owner satisfaction, with a low incidence of adverse events.

\section{Methods}

\section{Overview}

This study protocol was approved and followed the ethical principles of CEUA - UNISA and Plataforma Brasil. The owners or handlers responsible for care of the animals gave written informed consent for each dog to participate in the study.

This was a prospective study conducted from May 2019 to July 2020, with dogs followed for 30 days after treatment. The dogs enrolled were patients from 2 veterinary clinics specializing in veterinary dermatology: Cãopanheiro (Sorocaba-SP) and Dermatoclínica (São Paulo-SP). History was documented for each dog, and each dog was given a complete physical examination. Dermatological examinations were performed when appropriate, including parasitological examination of skin scrapings, cytology for bacteria and/or yeast, microbiological cultures, or skin biopsies.

\section{Inclusion criteria}

Dogs of any breed or sex, weighing at least $2 \mathrm{~kg}$, and diagnosed with AD according to meeting at least 6 of the 8 Favrot's 2010 criteria [5] and having a minimum CADESI-4 of 20 and a minimum VAS score of 7 were eligible to be enrolled in the study. Dogs diagnosed with cutaneous adverse food reactions remained on the same diet throughout the study. All dogs were free of ectoparasites on day 0 and prevention of new infestations was maintained throughout the study.

Dogs currently receiving other treatments for $A D$ must have discontinued those treatments as follows before receiving treatment in the study: oclacitinib: 3 days; oral corticosteroids or cyclosporine: 4 weeks;antimicrobial therapy;. Dogs with superficial pyoderma were able to use topical antiseptic formulations for 15 days before and into the study period as needed.

\section{Exclusion criteria}

Exclusion criteria included the following:

- signs of intolerance to lokivetmab

- treatment with allergen-specific immunotherapy

- history or diagnosis with malignant neoplasms

- treatment with corticosteroids (systemic or topical) during the study

- immunization with attenuated vaccines $<2$ weeks

- age less than 1 year 
- pregnant or lactating females

- concomitant diseases during the study with a need for specific treatments

\section{Treatment administration}

Dogs were individually weighed on day 0 for determination of the correct dose. Dogs received lokivetmab at $2 \mathrm{mg} / \mathrm{kg} \mathrm{SC}$ and were kept under observation for 30 minutes after treatment. Owners were subsequently contacted by telephone or e-mail 2 and 14 days after their dog was treated. On day 30 , the animals were returned to the clinic for follow-up evaluation by the veterinarian.

\section{Evaluations}

Treatment success was based on VAS for pruritus, CADESI-4 scores for skin lesions, and Owner Global Assessment of Treatment Efficacy (OGATE) for owner perception of efficacy. Forms for these assessments were sent to owners by email for completion. On day 30, VAS, CADESI-4, and OGATE forms were filled out by the owners and veterinarian at the time of the dog's post-treatment evaluation.

Treatment success was defined as a $50 \%$ or greater reduction in the VAS and a $\geq 2$-cm reduction in the baseline VAS scale and a decrease of $\geq 50 \%$ of CADESI- 4 by day 30 from that recorded on day 0 .

\section{Statistical analysis}

Initially, all variables were evaluated by descriptive techniques. Analysis of quantitative variables was performed by calculating mean, standard deviation (SD), and median. For qualitative variables, absolute and relative frequencies were calculated. Normality of the data was determined by the KolmogorovSmirnov test, and homogeneity between proportions was determined by Fisher's exact test. Comparisons of data collected during the study were made using the non-parametric test of Friedman and Wilcoxon. Also, for the comparison of proportions observed, the McNemar non-parametric test was used [10]. Calculations were performed using SPSS 17.0 for Windows. The level of significance was $5 \%$.

\section{Abbreviations}

AD, atopic dermatitis; CADESI-04, Canine Atopic Dermatitis Extent and Severity Index; IL-31, interleukin-31; mAb, monoclonal antibody; OGATE, Owner Global Assessment of Treatment Efficacy; SC, subcutaneous; SD, standard deviation; VAS, visual analog scale

\section{Declarations}

\section{Acknowledgments}

The authors thank the owners for providing their dogs to participate in this evaluation and for their cooperation to observe and record conditions of the dogs during the study. We also thank Kathleen 
Newcomb, Blythewood Consulting, LLC, Nathalie, Virginia, USA for editorial support in the preparation of this manuscript.

\section{Funding}

All lokivetmab (Cytopoint ${ }^{\circledR}$ ) was provided by Zoetis Industria de Produtos Veterinarios Ltda, Sao Paulo, Brazil.

\section{Author Contributions}

$\mathrm{RL}$ and MLC designed the study, supervised research, performed the sampling and statistical analyses, drafted and edited the manuscript. VM contributed to design the study. DB, CP, JD and VM performed sampling analysis. JF-M revised and edited the manuscript. All the authors have read and approved the manuscript, and agreed to be accountable for their own contributions in ensuring that questions related to the accuracy or integrity of any part of the work are appropriately investigated and resolved.

\section{Ethics declarations}

This study protocol was approved and followed the ethical principles of CEUA - UNISA, Plataforma Brasil and is reported in accordance with ARRIVE guidelines 2.0. The owners or handlers responsible for care of the animals gave written informed consent for each dog to participate in the study. Treatments were administered by veterinarians specializing in treatment of dermatological conditions in dogs and other animal species.

\section{Availability of Date and Materials}

The datasets analysed during the current study are not publicly available due to the presence of additional data that were not used in the present study. Probably it will be useful for future publications, but are available from the corresponding author on reasonable request.

\section{Consent to publish}

Not applicable

\section{Competing interests}

The authors declare no competing interests

\section{References}

1. Hensel P, Santoro D, Favrot C, Hill P, Griffin C: Canine atopic dermatitis: detailed guidelines for diagnosis and allergen identification. BMC Vet Res 2015, 11:196.

2. Bernstein JA, Tater K, Bicalho RC, Rishniw M: Hair and saliva analysis fails to accurately identify atopic dogs or differentiate real and fake samples. Vet Dermato/ 2019. 
3. Bizikova P, Santoro D, Marsella R, Nuttall T, Eisenschenk MN, Pucheu-Haston CM: Review: Clinical and histological manifestations of canine atopic dermatitis. Vet Dermato/ 2015, 26(2):79-e24.

4. Michels GM, Ramsey DS, Walsh KF, Martinon OM, Mahabir SP, Hoevers JD, Walters RR, Dunham SA: A blinded, randomized, placebo-controlled, dose determination trial of lokivetmab (ZTS-00103289), a caninized, anti-canine IL-31 monoclonal antibody in client owned dogs with atopic dermatitis. Vet Dermato/ 2016, 27(6):478-e129.

5. Favrot C, Steffan J, Seewald W, Picco F: A prospective study on the clinical features of chronic canine atopic dermatitis and its diagnosis. Vet Dermatol 2010, 21(1):23-31.

6. Merck Veterinary Manual. Available at https://www.merckvetmanual.com/integumentarysystem/atopic-dermatitis/canine-atopic-dermatitis. Accessed March 21, 2021.

7. Santoro D, Marsella R, Pucheu-Haston CM, Eisenschenk MN, Nuttall T, Bizikova P: Review: Pathogenesis of canine atopic dermatitis: skin barrier and host-micro-organism interaction. Vet Dermato/ 2015, 26(2):84-e25.

8. Rybnicek J, Lau-Gillard PJ, Harvey R, Hill PB: Further validation of a pruritus severity scale for use in dogs. Vet Dermatol 2009, 20(2):115-122.

9. Gonzales AJ, Humphrey WR, Messamore JE, Fleck TJ, Fici GJ, Shelly JA, Teel JF, Bammert GF, Dunham SA, Fuller TE et al: Interleukin-31: its role in canine pruritus and naturally occurring canine atopic dermatitis. Vet Dermatol 2013, 24(1):48-53 e11-42.

10. Fleck TJ, Bammert G, Shelly J, Fici G, Walters RR, Mahabir S, Michels GM, Rugg JJ, Martinon O, Dunham S: Identification and characterization of ATS-00103289, a monoclonal antibody neutralizing interleukin-31-mediated pruritus, in beagle dogs. Vet Dermatol 2015, 26:147.

11. Gortel K: An embarrassment of riches: An update on the symptomatic treatment of canine atopic dermatitis. Can Vet J 2018, 59(9):1013-1016.

12. Walters RR, Boucher JP, Paquette JA, Birkholz PJ, Mahabir S, Dunham S, Fleck TJ: Laboratory dose titration efficacy study of ZTS-00103289, a caninized anti-interleukin-31 monoclonal antibody, in a canine model of IL-31 induced pruritus (Abstract). Vet Dermatol 2015, 26:145.

13. Moyaert H, Van Brussel L, Borowski S, Escalada M, Mahabir SP, Walters RR, Stegemann MR: A blinded, randomized clinical trial evaluating the efficacy and safety of lokivetmab compared to ciclosporin in client-owned dogs with atopic dermatitis. Vet Dermatol 2017, 28(6):593-e145.

14. Michels GM, Walsh KF, Kryda KA, Mahabir SP, Walters RR, Hoevers JD, Martinon OM: A blinded, randomized, placebo-controlled trial of the safety of lokivetmab (ZTS-00103289), a caninized anticanine IL-31 monoclonal antibody in client-owned dogs with atopic dermatitis. Vet Dermato/2016, 27(6):505-e136.

15. CVMP assessment report for CYTOPOINT. European Medicines Agency. Available at https://www.ema.europa.eu/en/documents/assessment-report/cytopoint-epar-public-assessmentreport_en.pdf. Accessed March 21, 2021.

16. Cytopoint Package Insert (USA). Zoetis, Inc. Available at https://www.zoetisus.com/products/dogs/cytopoint/assets/resources/2019/cytopoint-approved- 
package-insert.pdf,

17. Olivry T, DeBoer DJ, Favrot C, Jackson HA, Mueller RS, Nuttall T, Prelaud P, International Task Force on Canine Atopic D: Treatment of canine atopic dermatitis: 2010 clinical practice guidelines from the International Task Force on Canine Atopic Dermatitis. Vet Dermatol 2010, 21 (3):233-248.

18. Buzz Sharer. 30 most popular dog breeds in Brazil. Available at https://buzzsharer.com/blogpost/37633/ . Accessed March 21, 2021

19. Olivry T, Bensignor E, Favrot C, Griffin CE, Hill PB, Mueller RS, Plant JD, Williams HC, International Committee of Allergic Diseases of A: Development of a core outcome set for therapeutic clinical trials enrolling dogs with atopic dermatitis (COSCAD'18). BMC Vet Res 2018, 14(1):238.

\section{Figures}

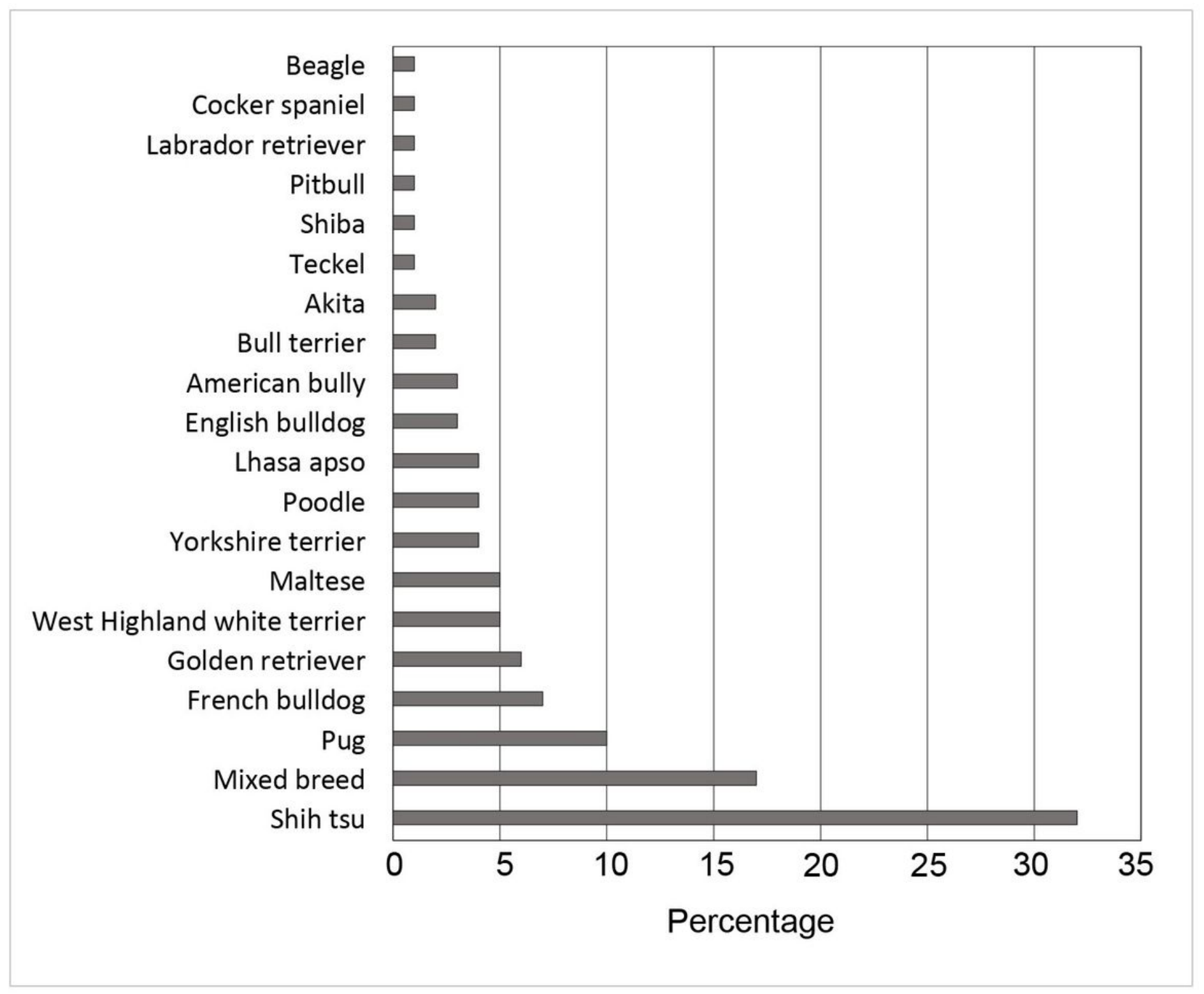

Figure 1 
Frequency of dog breeds diagnosed with atopic dermatitis and treated with lokivetmab day $0(\mathrm{~N}=110)$

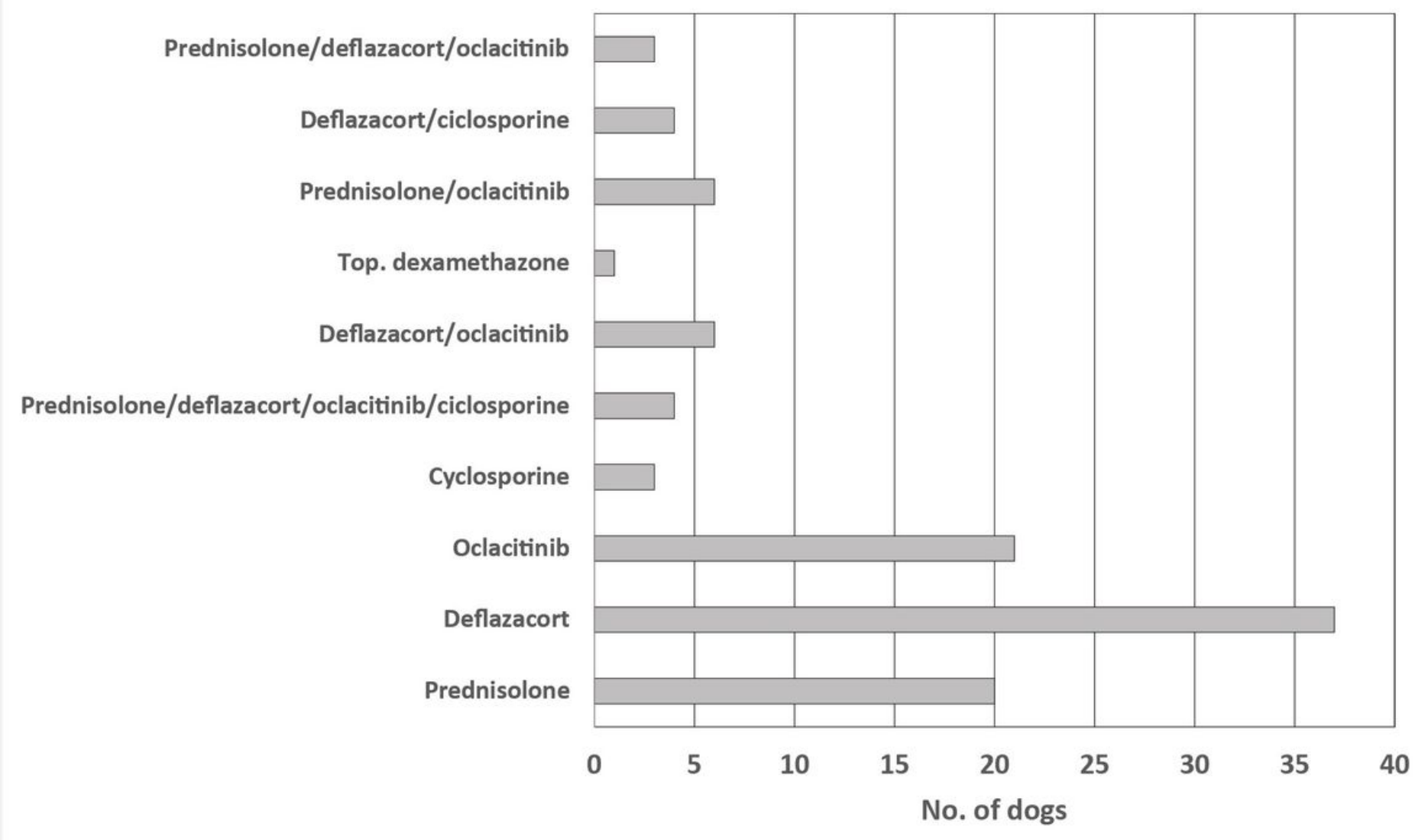

\section{Figure 2}

Previous treatments for client-owned dogs enrolled in study of lokivetmab against atopic dermatitis $(\mathrm{N}=$ 106) 


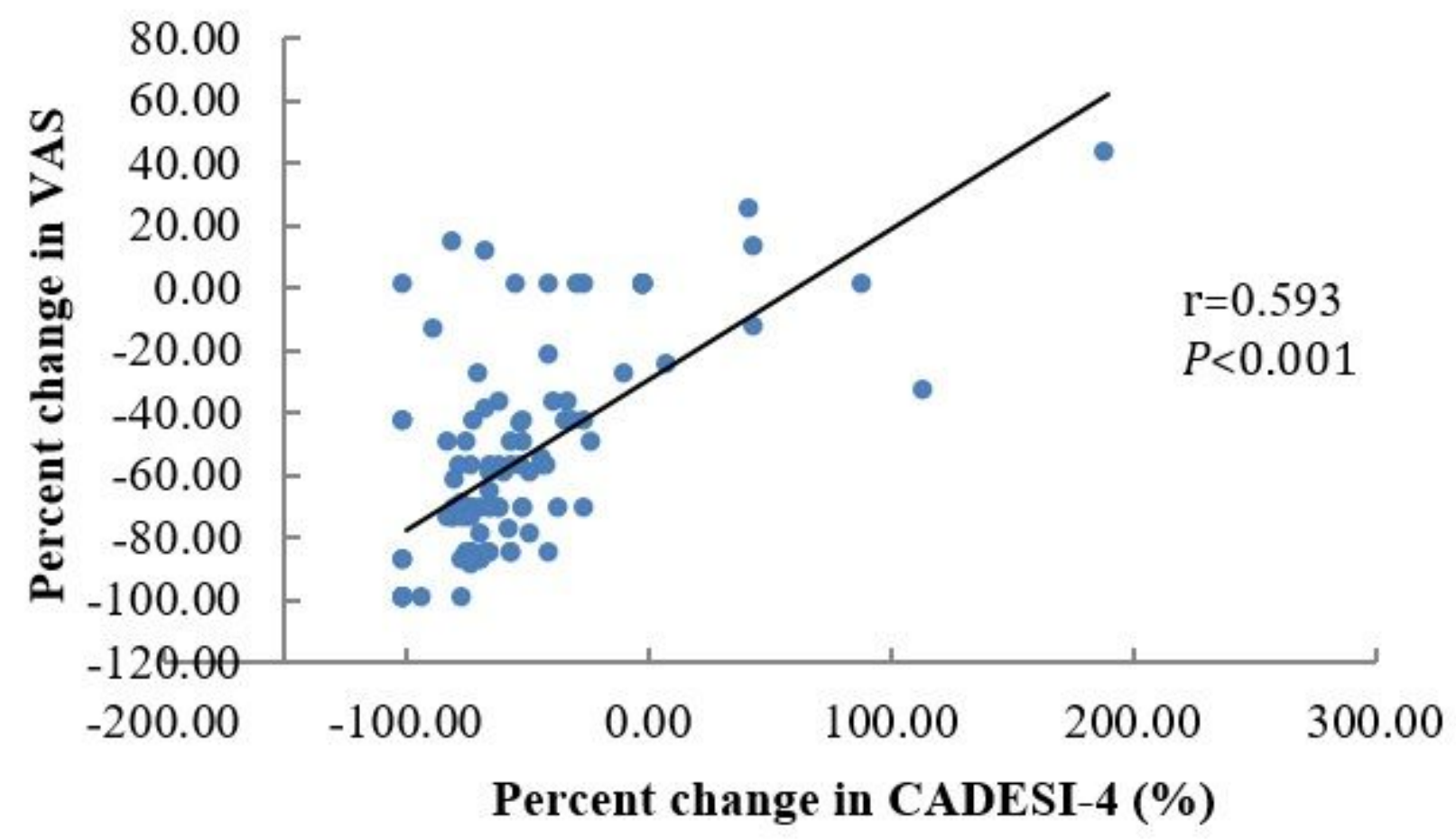

Figure 3

Spearman correlation between VAS and CADESI-4 of patients with AD treated with lokivetmab $(N=106)$

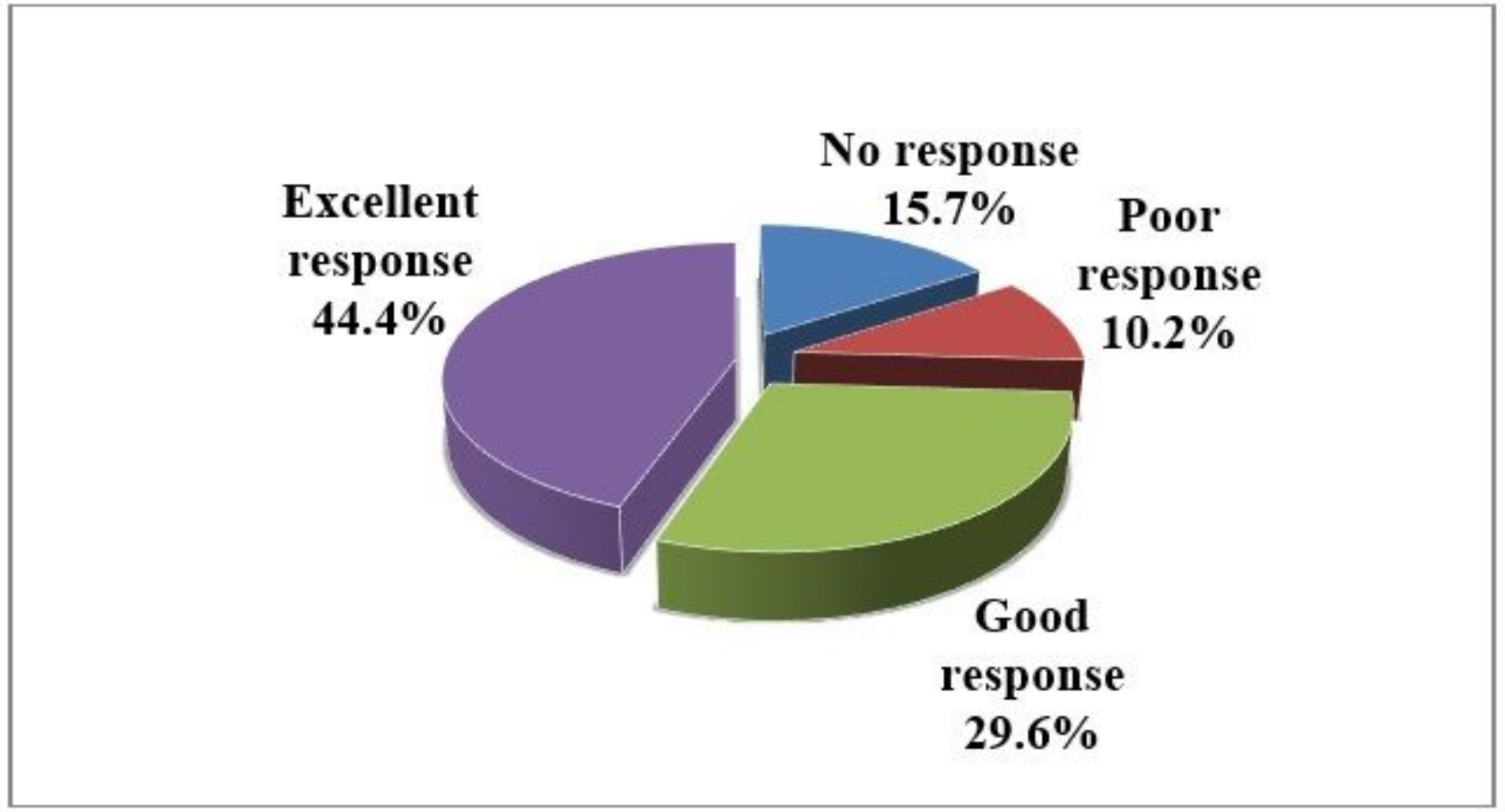

Figure 4

Distribution of responses provided by owners regarding their perception of treatment efficacyn in dogs with $A D$ treated with lokivetmab day $0(\mathrm{~N}=106)$. 